\title{
LA TRANSFORMACIÓN DE LA DOCTRINA DE LA VIRTUD MORAL EN EL PENSAMIENTO DE JuAN DUNS Escoto
}

\author{
Emiliano Javier Cuccia ${ }^{l}$
}

\begin{abstract}
RESUMEN: En su comentario a la distinción 33 del Tercer Libro de las Sentencias, Juan Duns Escoto desarrolla su doctrina referida al sujeto de las virtudes morales, mediante la cual establece la voluntad como única sede posible de las mismas. En el presente trabajo se intentará mostrar que esta determinación, por un lado, es consecuencia de la previa postulación de la voluntad como única potencia moral del hombre y que, por otro, implica una fuerte debilitamiento del rol e importancia de la virtud para la perfección moral.
\end{abstract}

PALABRAS CLAVE: Juan Duns Escoto. Virtud moral (sujeto). Voluntad. Filosofía moral.

\section{INTRODUCCIÓN}

En la actualidad no es posible desconocer la importancia que la labor intelectual de Juan Duns Escoto ha tenido en el desarrollo de la historia de la Filosofía. Sin lugar a dudas, se trata de uno de los máximos representantes de la Escolástica de finales del siglo XIII y principios del XIV, y una eximia figura de la Escuela Filosófica Franciscana. Sus escritos, tanto teológicos como filosóficos, han alimentado y alimentan aún hoy discusiones referidas a la naturaleza divina, al conocimiento humano y a la polémica sobre los universales, entre otras.

Sin embargo, una dimensión poco explorada de su pensamiento la constituyen sus opiniones referidas a la moral y, particularmente, a las virtudes y vicios. Como prueba de ello puede citarse la relativamente poca cantidad de estudios y artículos que intenten dar cuenta del pensamiento del Doctor Sutil en este ámbito.

Afortunadamente, esta situación parece comenzar a revertirse a partir de la publicación, en edición crítica, de sus comentarios al libro III de las Sentencias en los cuales desarrolla la mayor parte de sus ideas acerca de la

\footnotetext{
${ }^{1}$ Prof. Lic. Facultad de Filosofía y Letras, UNCuyo - CONICET (Argentina) - E-mail: ecuccia@ gmail.com
} 
vida moral. Concretamente, poseen una gran importancia sus glosas a la distinción 33 referidas a la determinación del sujeto de las virtudes morales, ${ }^{2}$ por dos motivos. En primer lugar, porque es uno de los pocos lugares donde el Maestro Escocés realiza un estudio detenido acerca de estos hábitos que, según la mayoría de los maestros escolásticos, eran los responsables de perfeccionar al hombre y hacer buena su obra (e.g. KENT, 2003, p. 355). ${ }^{3} \mathrm{Y}$ en segundo lugar, porque su doctrina supone una transformación del esquema tradicional referido al sujeto de tales perfecciones.

Por más que esta modificación parecería ser, a primera vista, un asunto de poca importancia, dentro de la óptica escolástica conlleva una nueva determinación de lo que sea la virtud moral y de lo que sea una persona virtuosa (e.g. KENT, 1995, p. 199). Así, se intentará probar en el presente artículo que el cambio introducido por Escoto en el sujeto de las virtudes morales implica tanto la modificación profunda de la naturaleza de dichos hábitos, como la de su rol e importancia para la perfección moral.

Para lograr este cometido en primer lugar se sintetizará la doctrina contenida tanto en Ordinatio III, 33 como en Lectura III, 33. En segundo lugar, se mostrará hasta qué punto influyó en la génesis de tales ideas la consideración de la voluntad como única potencia moral. Y, finalmente, se constatará la magnitud del impacto que ejercieron en la consideración del rol y naturaleza de la virtud moral.

\section{I- EL SUJETO DE LA VIRTUD MORAL}

En sus comentarios ${ }^{4}$ a la distinción 33 del tercer libro de las Sentencias, Duns Escoto plantea un esquema diferente al tradicional en lo que respecta a la determinación del sujeto de las virtudes morales. Si bien el Maestro

\footnotetext{
${ }^{2}$ Se hace referencia a los textos correspondientes a la Ordinatio III, 33 y la Lectura III, 33, publicados respectivamente en los tomos X y XXI de la Edición Vaticana de la Opera Omnia del Doctor Sutil, a cargo de la Comisión Escotista. B. IOANNIS DUNS SCOTUS. Opera omnia. v.10 (Ordinatio III, dist. 26-40). B. Hechich, B. Huculak, J. Percan, and S. Ruiz de Loizaga (Ed). Città del Vaticano: Typis Vaticanis, 2007 y v.21 (Lectura III, dist. 18-40). B. Hechich, B. Huculak, J. Percan, S. Ruiz de Loizaga, and C. Saco Alarcón (Ed). Città del Vaticano: Typis Vaticanis, 2003. Todas las referencias a los textos de Juan Duns Escoto efectuadas en el presente trabajo pertenecen a las ediciones citadas.

${ }^{3}$ Cf. TOMÁS DE AQUINO, Sententia Libri Ethicorum, II, 6, nn. 2 et 3; De virtutibus in communi a5, in corp.; ARISTÓTELES, Ética Nicomáquea, II, 51106 a 15-17.

${ }^{4} \mathrm{Si}$ bien los argumentos analizados y transcriptos en este artículo son extraídos, en su mayoría, del texto de la Ordinatio, debe decirse que en su Lectura Duns Escoto defiende prácticamente la misma doctrina. Para poner de manifiesto tal correspondencia entre una y otra obra, cuando en el artículo
} 
Franciscano mantiene a la inteligencia como sujeto de la prudencia y a la voluntad como sujeto de la justicia, al momento de definir el sujeto de la fortaleza y de la templanza niega que ambas puedan residir, respectivamente, en los apetititos irascible y concupiscible del hombre. Por el contrario, ambas son consideradas como virtudes que anidan en la voluntad. Para defender su posición, aduce numerosos argumentos que se sintetizan a continuación.

En primer lugar, Duns Escoto sostiene que las virtudes morales deben residir necesariamente en la voluntad apoyándose en la definición que Aristóteles brinda de tales perfecciones en el libro II de la Ética Nicomáquea: "[...] la virtud moral es un hábito electivo".5 Quizá sorprenda notar que esta definición también es utilizada como argumento por otros pensadores -como Tomás de Aquino ${ }^{6}$, por ejemplo- sin que esto sea un obstáculo para que coloquen la fortaleza y la templanza en la apetencia sensible. La discrepancia se entiende si se cae en la cuenta de una novedad capital que introduce el Doctor Sutil al momento de considerar la naturaleza del acto de elección perfeccionado por la virtud moral. Mientras que para Tomás la elección es un acto fruto de la interacción entre cualquier apetito (sensible o intelectual) y la inteligencia, ${ }^{7}$ para Duns Escoto, la elección es un acto del apetito conciliativo, es decir, de la voluntad y de la razón conjuntamente, aunque, de hecho, corresponda más a la voluntad puesto que es esta última la que opera, si bien lo hace con el juicio presupuesto de la razón. Así, como el hábito es propio de aquella potencia a la cual pertenece la operación que perfecciona, entonces el hábito moral es, de suyo, propio de la voluntad. ${ }^{8}$

se apele a algún argumento presente en una de ellas, se dejará asentado también en el pie de página la referencia completa al pasaje paralelo en la otra.

${ }^{5}$ Cf. ARISTÓTELES. Ética Nicomáquea II, c. 6, 1106 b 36 - 1107 a 1.

${ }^{6}$ Se hace especial hincapié en la doctrina de Tomás de Aquino ya que Escoto desarrolla su comentario como una crítica frente a las ideas defendidas por el Angélico. Cf. DUNS ESCOTO, Ordinatio III, dist. 33, q. un., n. 10 (lin. $40-44$ ).

${ }^{7}$ Para una explicación más amplia de lo que Tomás de Aquino afirma acerca de la elección y de la participación en ella del apetito sensible cf. TOMÁS DE AQUINO, In III Sent., dist. 33, q. II, art. 4, sol. 2.

${ }^{8}$ Cf. DUNS ESCOTO, Ordinatio III, dist. 33, q. un., n. 7 (lin. 27 - 32): “Virtus est habitus electivus' ex definitone sua, ex II Ethicorum, -electio autem est actus vel voluntatis vel rationis, secundum Philosophum VI Ethicorum: 'est enim vel appetitus consiliativus vel' etc; haec pertinent ad voluntatem, quae operatur praesupposita cognitione rationis. Habitus est illius potentiae cuius est per se illa operatio; ergo habitus moralis est per se ipsius voluntatis." Cf. también Lectura III, dist. 33, q. un., n. 9 (lin. $43-45)$. 
En segundo lugar, el Doctor Sutil niega la posibilidad de que alguna virtud moral pueda existir en el apetito sensible. El motivo alegado consiste en que la virtud es, por sí misma, principio de acciones dignas de encomio. Pero la alabanza y el encomio se le otorgan sólo a aquel que obra de un modo propiamente humano, y lo que es propio del hombre y lo distingue de los demás animales no es la parte sensible sino la parte intelectual. ${ }^{9}$ Por lo tanto, si la virtud es considerada como principio de acciones encomiables debe residir en aquella parte del hombre que le es propia, o sea, la intelectiva; y no en la que comparte con los animales, esto es la sensible. ${ }^{10}$ Así, la virtud moral no puede existir en la apetencia sensible simplemente porque dicho apetito no participa propiamente de la racionalidad sino de un modo accidental. En este sentido, para Duns Escoto, la apetencia sensible en el hombre no parece presentar diferencias sustanciales con la propia del animal.

En tercer lugar, la consideración de la voluntad como sujeto de las virtudes morales se asume como necesaria a raíz del análisis de la naturaleza de dicha potencia. En efecto, el teólogo franciscano considera que no sólo la justicia sino también la fortaleza y la templanza deben residir en la voluntad por dos motivos. En primer lugar, por el carácter libre que posee dicha potencia, $y$, en segundo lugar, a causa de la evidente superioridad que la misma presenta frente a la sensibilidad humana.

Al hablar del carácter libre de la voluntad, el Beato se refiere a que la voluntad es indeterminada, y esto no sólo en lo que respecta a la inclinación hacia cosas opuestas, sino también en el modo de actuar: la voluntad puede obrar rectamente o no. Por consiguiente, necesita algo que la incline decididamente para obrar de un modo recto, y ésta es la función de la virtud moral: dirigir hacia el bien la acción de aquellas potencias que, de suyo, pueden actuar correcta o incorrectamente. Y como toda virtud moral, en tanto hábito, reside en aquella potencia cuya acción perfecciona, entonces la virtud moral

\footnotetext{
${ }^{9}$ Cf. DUNS ESCOTO, Ordinatio III, dist. 33, q. un., n. 28 (lin. 186 - 191): "illud enim magis indiget dispositione aliqua respectu cuius habet actionem in sua potestate quam respectu alterius, quia si non haberet in potestate, non sibi imputaretur nec ad laudem nec ad vituperium quando ageret vel qualiter. Sed quia in potestate sua habet, laudabiliter vel vituperabiliter agit; et ideo eget illo principio per quod possit laudabiliter operari: tale ponitur virtus. Cf. también Lectura III, dist. 33, q. un., n. 43 (lin. $229-232$ ).

${ }^{10}$ Cf. DUNS ESCOTO, Ordinatio III, dist. 33, q. un., n. 29 (lin. 192 - 194): "Videtur etiam mirabile quod illud propter quod laudatur homo in operando, sit praecise in eo secundum illud quod est commune sibi et brutis."
} 
debe residir en la voluntad. ${ }^{11}$ No obstante, debe aclararse, tal como el mismo Doctor Sutil lo hace, que esta indeterminación de la voluntad no implica para ella una imperfección sino que es una consecuencia de su ilimitación, la cual se constituye como su perfección propia. ${ }^{12}$ Por ello, la virtud moral no suprime el carácter ilimitado de la voluntad sino que la asiste en su acción, como la causa segunda a la causa primera, ayudándola a obrar según el modo recto pero sin eliminar su libertad, es decir, su capacidad de elegir entre contrarios. ${ }^{13}$

Por otro lado, la consideración de la superioridad de la voluntad sobre las potencias sensibles demuestra la necesidad de que la fortaleza y la templanza existan en la voluntad puesto que, si se admite que la misión de dichas virtudes consiste en moderar las pasiones, esto lo puede cumplir la voluntad de un mejor modo que la apetencia sensible por dos motivos. Primero, porque, según lo que afirma San Agustín en De civitate Dei, ${ }^{14}$ las pasiones están en la voluntad. Por consiguiente, si a raíz de la moderación de las pasiones la virtud es puesta en lo supremo de aquella potencia en la cual está la pasión, se sigue que en la parte suprema del alma, a saber la voluntad, están algunas pasiones y que allí son puestas las virtudes. ${ }^{15}$

Segundo, porque la moderación de las pasiones puede ser pensada doblemente, o bien de lo existente o bien de lo futuro. Pero de cualquier

\footnotetext{
${ }^{11}$ Cf. Duns Escoto, Ordinatio III, dist. 33, q. un., n. 22 (lin. 128 - 136): “...voluntas est indeterminata ad opposita, non tantum ad opposita obiecta, sed ad oppositos modos agendi, scilicet recte et non recte; ergo indiget aliquo determinativo inclinante ad recte agendum, et illud erit virtus. Consequen-tia patet: haec est enim sola necessitas ponendi virtutes in potentiis, ut per ipsas regulentur potentiae, quae de se possunt recte et non recte agere. Probatio antecedentis, quia voluntas potest eligere quodcumque sibi ostensum in quo ostenditur ratio sui primi obiecti; sed in eliciendo talia, potest non recte agere." Cf. también Lectura III, dist. 33, q. un., n. 32 (lin. 146 - 149).

12 Cf. DUNS ESCOTO, Ordinatio III, dist. 33, q. un., n. 30 (lin. 200 - 201): “...nec ista indeterminatio est ex imperfectione activi, sed ex illimitatione, quae est perfectio activi..." Cf. también Lectura III, dist. 33, q. un., n. 43 (lin. 233 - 243).

${ }^{13}$ Cf. DUNS ESCOTO, Ordinatio III, dist. 33, q. un., n. 32 (lin. 210 - 215): “...licet voluntas ex libertate sua posset se determinare in agendo, tamen ex actione est receptiva alicuius habilitatis derelictae, inclinantis ad similem actionem; illa enim unica determinatio eius non est per formam naturalem (qualis est in igne ad operandum), sed est ex libera actione quae procedit a potentia indetermi nata, et ita determinabili per habitum.” También, 33, 219 - 220: “... habitus autem, si est causa substantiae actus, est causa secunda respectu voluntatis.” Cf. también Lectura III, dist. 33, q. un., n. 45 (lin. 253 - 266).

${ }^{14}$ Cf. AGUSTÍN DE HIPONA, De civitate Dei XIV cap. 5 y 6.

${ }^{15}$ Cf. DUNS ESCOTO, Ordinatio III, dist. 33, q. un., n. 34 (lin. 223 - 227): “Tum quia in voluntate sunt passiones, secundum Augustinum XIV De civitate cap. 5 et 6 ; et propter hoc, si propter actionem et passionem ponenda sit virtus in illa potentia in qua est passio et actio voluntatis, sequitur quod cum passiones aliquae sint in suprema parte animae, quod ibi ponantur virtutes." Cf. también Lectura III, dist. 33, q. un., n. 46 (lin. 270-281).
} 
modo que sea pensada, dicho acto compete más a la voluntad que al apetito sensitivo. ${ }^{16}$ Pues acerca de la pasión que existe, si es posible que sea moderada, la voluntad, que actúa libremente, puede moderarla mejor que el apetito sensitivo, el cual padece a causa del objeto. ${ }^{17} \mathrm{Y}$ acerca de la pasión que aún no existe pero que puede llegar a existir, sólo la voluntad puede moderarla puesto que dicha acción se realiza o bien evitando el objeto que pueda deleitar inmoderadamente la potencia, o bien aceptando sólo los objetos que deleitan moderadamente. Y ambas operaciones corresponden únicamente a la voluntad y no al apetito sensible, puesto que sólo la voluntad puede realizar un acto recto acerca de lo futuro en la medida en que su facultad cognitiva correspondiente, esto es la razón, puede juzgar acerca del porvenir, y no así el sentido. ${ }^{18}$

Mediante los citados argumentos -y algunos otros no citados aquíDuns Escoto da por demostrado que sólo la voluntad puede ser sujeto de las virtudes morales.

\section{II- LA VOLUNTAD COMO RACIONAL Y LIBRE}

Dentro de la argumentación referida al sujeto de las virtudes morales, se percibe una destacada valoración de la voluntad frente a las otras potencias del hombre. Dicha estimación, que ha sido advertida por numerosos especialistas como una constante de la filosofía moral escotista (e.g. INGHAM, 1990a, p. 183; INGHAM, 1990b, p. 91; KENT, 2003, p. 356; WILLIAMS, 2012, p. 3) no es más que el corolario lógico de una de las tesis más radicales defendidas por el Doctor Sutil: la consideración de la voluntad como única potencia racional y libre del hombre.

\footnotetext{
${ }^{16}$ Cf. DUNS ESCOTO, Ordinatio III, dist. 33, q. un., n. 36 (lin. 240 - 242): “Quocumque istorum modorum intelligatur moderatio passionis, magis potest competere voluntati quam appetitui sensitivo, si passio inest vel inerit illi appetitui sensitivo...”

${ }^{17}$ Cf. DUNS ESCOTO, Ordinatio III, dist. 33, q. un., n. 36 (lin. 243 - 245): "Nam de passione inexsistente - si possibile est eam imminui voluntas potest eam imminuere, quae libere agit, magis quam appetitus sensitivus qui patitur ab obiecto vel coagit sibi quantum potest." Cf. también Lectura III, dist. 33, q. un., n. 48 (lin. 295 - 302).

${ }^{18}$ Cf. DUNS ESCOTO, Ordinatio III, dist. 33, q. un., n. 36 (lin. 252 - 256): "Si intelligatur de passione quae immoderata debet praecaveri et moderata assumi, satis videtur planum quod circa futurum magis potest voluntas habere actum rectum quam appetitus sensitivus, quia cognitiva voluntatis -quae est ratio- potest de futuris consiliari, non sic sensus." Cf. también Lectura III, dist. 33, q. un., n. 48 (lin. 295 - 302).
} 
Esta teoría es desarrollada con mayor detenimiento en la cuestión 15 de su comentario al libro IX de la Metafísica de Aristóteles. ${ }^{19}$ Allí establece que, si por potencia racional debe entenderse aquella que, manteniendo su carácter unitario, es capaz de elegir cualquier acción opuesta o, incluso, de abstenerse de la acción, entonces ni la sensibilidad ni la propia inteligencia en sí misma pueden ser consideradas racionales sino sólo la voluntad. ${ }^{20}$ Quizá sorprenda observar que se le niegue a la inteligencia el carácter propiamente racional, pero es necesario comprender a qué responde esta definición tan categórica.

Duns Escoto establece que hay dos formas de determinar el carácter de una potencia: o bien con relación a su propia acción, o bien con relación a las acciones de las potencias sobre las cuales ejerce su causalidad. ${ }^{21}$ La primera de estas formas de determinación es más esencial y, según ella, la inteligencia opera como una facultad natural porque realiza su acto propio, que es el conocimiento, de un modo necesario: la inteligencia no puede elegir no conocer, ${ }^{22}$ así como tampoco lo pesado puede elegir no caer, ni el fuego puede elegir no calentar. En cambio, según la otra forma de determinación, la inteligencia podría ser considerada racional sólo en la medida en que su acto de conocimiento es una condición previa para el acto de una potencia racional, como es la voluntad. ${ }^{23}$ De esta manera, el único acto propiamente racional realizado por el hombre sería el acto electivo de la voluntad, el cual, si bien necesita del conocimiento de los opuestos brindado por la inteligencia, es absolutamente libre en la medida en que es fruto de la autodeterminación de la voluntad. Así, al ser éste el único acto racional y libre del hombre, es también el único acto moral. ${ }^{24}$

\footnotetext{
${ }^{19}$ La fuente utilizada corresponde a la traducción de Allan B. Wolter, editada por William A. Frank (apud: Wolter, 1997). Existe una traducción al español realizada por Cruz González Ayesta: JUAN DUNS ESCOTO. Naturaleza y voluntad: Quaestiones super libros Metaphysicorum Aristotelis, IX, q. 15. Introducción, traducción y notas de Cruz González Ayesta. Cuadernos de Anuario Filosófico Serie Universitaria 199. Pamplona: EUNSA, 2007.

${ }^{20}$ Cf. DUNS ESCOTO, Quaestiones super libros Metaphysicorum Aristotelis, IX, q. 15. (apud: Wolter, 1997, p. 137).

${ }^{21}$ Cf. DUNS ESCOTO, Quaestiones super libros Metaphysicorum Aristotelis, IX, q. 15. (Wolter, 1997, p. 141).

${ }^{22}$ Cf. DUNS ESCOTO, Quaestiones super libros Metaphysicorum Aristotelis, IX, q. 15. (Wolter, 1997 p. 141).

${ }^{23}$ Cf. DUNS ESCOTO, Quaestiones super libros Metaphysicorum Aristotelis, IX, q. 15. (Wolter, 1997 p. 142)

${ }^{24}$ Para un análisis de la influencia que ejercieron sobre esta doctrina escotista tanto algunos artículos condenados por el obispo parisino Esteban Tempier en 1277 (particularmente los referidos a la libertad divina) como los planteos éticos de los teólogos franciscanos anteriores a Escoto cf. INGHAM, 1990b, p. 96, 97 y 101-103; WILLIAMS, 2012, p. 7 y 8; KENT, 1995)
} 
En este sentido, según el pensamiento del Doctor Sutil, la voluntad no puede ser la potencia apetitiva intelectual que busca naturalmente aquello que la inteligencia le muestra como bueno, tal como opinaban algunos de sus contemporáneos. ${ }^{25} \mathrm{Si}$ así fuese, entonces habría que negarle a la voluntad toda libertad y considerarla como una facultad que opera de un modo natural, lo cual pondría en jaque la responsabilidad del hombre frente a sus acciones. En efecto, toda acción pecaminosa debería atribuirse a una falla del intelecto ocurrida al captar el bien o en el momento de mostrarlo ante la voluntad. Y, cualquiera fuese el caso, se suprimiría inmediatamente la responsabilidad de la persona por su mala acción ya que habría operado similarmente a como lo haría si estuviese cegado por la ignorancia. ${ }^{26}$

Por el contrario, para Duns Escoto, la única forma de asegurar la libertad y responsabilidad del hombre en sus acciones específicas es considerando a la voluntad como una potencia eminentemente activa y no meramente pasiva de la acción de la inteligencia. Y, además, como la única potencia propiamente libre y racional en el hombre. Esto implica que la voluntad tenga absoluta soberanía con respecto a sus decisiones y que, lejos de tener que obedecer necesariamente a la razón, pueda contradecirla cuantas veces lo desee ya que se encontraría totalmente indeterminada hacia cualquiera de los opuestos y de los modos de obrar contrarios.

Las consecuencias de esta doctrina saltan a la vista: el factor central y determinativo de la vida moral lo constituye la operación de la voluntad (e.g. KENT, 2003, p. 356). Y si bien admite que la correspondencia de la voluntad con la recta razón es absolutamente necesaria para la bondad del acto moral, dicha concordancia depende exclusivamente de la autodeterminación de la voluntad. ${ }^{27}$ Así mismo, cualquier elemento externo que determinara a la voluntad atentaría contra la libertad de dicha potencia y amenazaría con suprimir la moralidad de la acción.

Por este motivo se entiende que Duns Escoto no pueda pensar para la virtud moral otro sujeto que no sea la voluntad: porque sólo esta potencia es el recinto de la moralidad al ser la única potencia libre, esto es indeterminada y autodeterminante.

\footnotetext{
${ }^{25}$ Como, por ejemplo, Godofredo de Fontaines (Cf. KENT, 1995, p. 141).

${ }^{26}$ Cf. DUNS ESCOTO, Lectura III, dist. 33, q. un., n. 34 (lin. 153 - 160).

${ }^{27}$ Cf. DUNS ESCOTO, Quodlibet, q. 18 (WOLTER, 1997, p. 170).
} 


\section{III- Rol DE LA VIRTUd EN Duns Escoto}

Tanto la afirmación de la voluntad como única potencia racional desarrollada por el fraile escocés, como la consideración limitada del sujeto de la virtud moral constituyeron un nuevo horizonte dentro del cual tanto la naturaleza de dicho hábito como el rol que debía cumplir dentro de la vida moral de la persona fueron resignificados.

En una postura que consideraba la apetencia sensible, junto con la voluntad, como posible sujeto de las virtudes morales, estas últimas cumplían la función de dirigir las potencias humanas racionales por participación (a saber, voluntad, apetito concupiscible y apetito irascible) hacia la armonía con la recta razón. Esta misión se consideraba absolutamente necesaria para la buena vida moral en la medida en que las potencias citadas, si bien estaban llamadas a participar de la racionalidad, podían, mediante alguno de sus actos, obrar en contra del bien indicado por la recta razón. Esto mismo es lo que argumenta, por citar un ejemplo, Tomás de Aquino acerca del apetito irascible y concupiscible:

[...] el irascible y el concupiscible no obedecen al mandato de la razón, por el contrario tienen sus propios motivos, con los cuales a veces repugnan a la razón: por lo cual, en el mismo libro [ $\mathrm{I}^{\circ}$ de la Política], el Filósofo dice que la razón gobierna el irascible y el concupiscible con 'gobierno político', por el cual, a saber, los [hombres] libres son gobernados, los cuales tienen, en cada uno, su propia voluntad. Y a causa de esto también corresponde que en el irascible y el concupiscible haya algunas virtudes, por las cuales sean bien dispuestos hacia el acto. ${ }^{28}$

En este sentido, el hombre moralmente perfecto era aquel que, mediante la virtud, lograba que todas sus potencias pasibles de racionalidad estuvieran bien dispuestas y hacia su acto recto, y esto de un modo estable y permanente. En otras palabras, la virtud cumplía un rol de crucial importancia para la excelencia moral: determinar las potencias mencionadas hacia la armonía con la recta razón. Por este motivo, el Aquinate podía afirmar con Aristóteles que la virtud era aquello que perfeccionaba al poseedor y volvía buena su obra. ${ }^{29}$ Así, la virtud implicaba la formación de un carácter que hacía, si no imposible, al menos muy difícil que la persona obrara de un modo contrario. (e.g. KENT, 2003, p. 352).

\footnotetext{
${ }^{28}$ Tomás de Aquino, Summa Theologiae. I-II q. 56, a.4 ad 3.

${ }^{29}$ Cf. TOMÁS DE AQUINO, Sententia Libri Ethicorum, II, 6, nn. 2 et 3, De virtutibus in communi a5, in corp.; ARISTÓTELES, Ética Nicomáquea, II, 51106 a 15-17.
} 
En cambio, la postura del Maestro Franciscano establece para la virtud moral un papel más modesto y secundario. Por un lado, en sus comentarios a las Sentencias, desecha la idea de que la virtud moral sea una condición necesaria para la bondad de la acción humana. Al menos esto es lo que parece desprenderse del siguiente pasaje de su Lectura: “[...] además, el hábito no es puesto para determinar la potencia, ni a causa de la sustancia del acto (porque [el acto] puede tenerse sin el hábito, ya que alguien se hace justo a causa del hacer [según] el II libro de la Ética)..." ${ }^{30}$ Además en Ordinatio III, 33 agrega que la voluntad puede obrar el acto recto y moralmente bueno sin el hábito. ${ }^{31}$ Bonnie Kent (2003, p. 359) destaca esta misma conclusión y enumera varios lugares donde Escoto sostiene que la virtud "[...] no afecta la sustancia moral de la acción" ya que "[...] un acto es moralmente bueno porque se conforma a todo lo que dicta la recta razón...” y no porque el agente posea la virtud moral correspondiente.

Por otro lado, Escoto sí admite que la virtud perfecciona al poseedor ya que, como afirma en la distinción 17 de su Ordinatio I, la voluntad "[...] opera menos perfectamente sin el hábito que con el hábito". ${ }^{22}$ Sin embargo, esta perfección que la virtud agrega nada tiene que ver con una determinación ${ }^{33}$ radical mediante la cual lograría que la voluntad quiera necesariamente el bien mostrado por la recta razón. Si así lo hiciera, entonces suprimiría el carácter libre de dicha potencia y anularía el valor de la acción humana.

Por el contrario, la virtud moral se comporta al modo de una causa segunda $^{34}$ que sólo colabora con la voluntad al hacer que ésta elija el bien de un modo casi imperceptible y placentero, ${ }^{35}$ pero sin hacer que este modo de comportamiento sea estable, permanente o necesario, ni agregar mayor bondad a la acción moral del sujeto. En otras palabras, así como la virtud

${ }^{30}$ DUNS ESCOTO, Lectura III, dist. 33, q. un., n. 35 (lin. 161 - 163).

${ }^{31}$ Cf. DUNS ESCOTO, Ordinatio III, dist. 33, q. un., n. 43 (lin. 282 - 283).

${ }^{32}$ Ordinatio I, d. 17, q. un., n. 43 Cit. en Ingham, 1990a, p. 190, nota 29.

${ }^{33}$ Debe decirse que Escoto, por momentos, declara que la virtud es puesta en la voluntad para determinar (Cf. Ordinatio III, dist. 33, q. un., n. 22 (lin. 128 - 131): "[...] voluntas [...] indiget aliquo determinativo inclinante ad recte agendum, et illud erit virtus"). Sin embargo, una lectura en profundidad de sus comentarios permite comprender que se refiere a una disposición inclinante y no a una determinación que obligue con necesidad.

${ }^{34}$ Cf. DUNS ESCOTO, Ordinatio III, dist. 33, q. un., n. 33 (lin. 216 - 220).

${ }^{35}$ Cf. DUNS ESCOTO, Ordinatio III, dist. 33, q. un., n. 77 (lin. 527 - 535). Bonnie Kent (2003, p. 361 y 362) caracteriza este rol de la virtud como un rol "psicológico". 
moral no es condición necesaria para la buena acción, tampoco es condición suficiente (e.g. WILLIAMS, 2012, p. 4; KENT, 2003, p. 359).

De este modo queda en evidencia que, dentro de la doctrina moral del Doctor Sutil, las virtudes pierden la centralidad que mantuvieron hasta ese momento en la ética occidental. La virtud no hace que el hombre sea permanentemente bueno ni hace buena su obra. ${ }^{36}$ Simplemente facilita -a modo de causa segunda o instrumental- el proceso de elección comandado por la voluntad, disminuyendo tanto el tiempo de deliberación como el riesgo de desvíos que podrían provocarse por el asalto de las pasiones desordenadas que se originan en el apetito sensible.

\section{ConClusión}

En la introducción se planteó como hipótesis la posibilidad de que la modificación en el sujeto de las virtudes morales propuesta por Duns Escoto tuviera implicancias más profundas que aquellas verificables a simple vista.

Luego del análisis realizado, ha quedado en evidencia que, si bien el Doctor Sutil dedica un minucioso examen a la cuestión de las virtudes morales, mediante el mismo introduce una importante transformación tanto en la naturaleza de dichos hábitos, como en la de su rol e importancia para la perfección moral. A partir de sus afirmaciones, la virtud moral no puede ser considerada como la perfección que hace bueno al hombre y a su obra, sino como una causa instrumental que sólo influye secundariamente en la sustancia de la buena acción, otorgando facilidad y velocidad en el proceso electivo.

A su vez, es posible verificar que tal doctrina depende fuertemente del énfasis otorgado a la elección voluntaria, al considerarla como el único acto propiamente moral del hombre. De esta manera, la cuestión de la excelencia moral estaría emparentada fundamentalmente con la autodeterminación de la voluntad en orden al cumplimiento de los mandatos de la recta razón (e.g. INGHAM, 1990a, p. 194), y no con la posesión de las virtudes morales.

Así, puede afirmarse que el planteo del Maestro Franciscano constituyó un punto de inflexión que supuso el debilitamiento de uno de los pilares principales sobre los que se sostuvo la ética hasta sus días: la posesión de las virtudes morales como condición indispensable para la excelencia moral.

${ }^{36}$ Cf. DUNS ESCOTO, Lectura III, dist. 33, q. un., n. 35 (lin. 161 - 164); y Ordinatio III, dist. 33, q. un., n. 43 (lin. $281-282$ ). 
Por otro lado, como algunos autores han destacado, sus ideas en éste ámbito conformaron un hito de gran importancia en el derrotero conceptual que llevó a un profundo cambio del paradigma ético entre la Edad Media y el Iluminismo (e.g. QUINN, 1996, p. 628; INGHAM, 1990a, p. 195; KENT, 2003, p. 366).

CUCCIA, Emiliano Javier. The transformation of the doctrine of moral virtue in the thought of John Duns Scotus. Trans/Form/Ação, Marília, v. 36, n. 2, p. 9-22, Maio./Ago, 2013.

ABSTRACT: In his commentary on distinction 33 in the Third Book of Lombard's Sentences, John Duns Scotus develops his doctrine concerning the subject of the moral virtues, according to which the will is the only possible seat of the virtues. This paper aims to show that this determination, on the one hand, results from the prior postulation of the will as the only moral power of man and, on the other, involves a serious weakening of the role and importance of virtue in moral perfection.

KEYWORDS: John Duns Scotus, Moral virtue, Will, Moral philosophy.

\section{REFERENCIAS}

AGUSTÍN DE HIPONA. La Ciudad de Dios. Edición bilingüe, traducción de Santos Santamarta del Río, O.S.A., y Miguel Fuertes Lanero, O.S.A., notas de Victorino Capanaga, O.S.A. Madrid: Biblioteca de Autores Cristianos, 1972.

ARISTÓTELES, Etica a Nicómaco. 7. edición bilingüe, traducción de Julián Marías y María Araujo, introducción y notas de Julián Marías. Madrid: Centro de Estudios Políticos y Constitucionales, 1999.

B. IOANNIS DUNS SCOTUS. Opera omnia. v.10 (Ordinatio III, dist. 26-40). B. Hechich, B. Huculak, J. Percan, and S. Ruiz de Loizaga (Ed). Città del Vaticano: Typis Vaticanis, 2007.

v. 21 (Lectura III, dist. 18-40). B. Hechich, B. Huculak, J. Percan, S. Ruiz de Loizaga, and C. Saco Alarcón (Ed). Città del Vaticano: Typis Vaticanis, 2004.

BUSA SJ, R. (auctore). Thomae Aquinatis Opera Omnia: cum hypertextibus in CD-ROM. Milano: Editoria Elettronica Editel, 1992. 
INGHAM, M. E. 'Ea quae sunt ad finem': Reflections on Virtue as Means to Moral Excellence in Scotist Thought. Franciscan Studies, v. 50, p. 177-195, 1990a.

. The Condemnation of 1277: Another Light on Scotist Ethics. Freiburger Zeitschrift für Philosophie und Theologie 37, p. 91-103, $1990 \mathrm{~b}$.

KENT, B. Virtues of the will: the transformation of ethics in the late thirteenth century. Washington, D.C.: The Catholic University of America Press, 1995.

. Rethinking Moral Dispositions: Scotus on the Virtues. In: WILLIAMS, T. (Ed.). The Cambridge Companion to Duns Scotus. Cambridge: Cambridge University Press, 2003, p. 352-376.

QUINN, P. 'Virtues of the Will: The Transformation of Ethics in the Late Thirteenth Century' by Bonnie Kent (Review). The Journal of Philosophy, v. 93, n. 12, Dec., p. 628$631,1996$.

TOMÁS DE AQUINO. Suma de Teología. Texto latino de la edición crítica leonina. Traducción y anotaciones por una comisión de PP. Dominicos presidida por el Excmo. y Rvdmo. Sr. Dr. Fr. Francisco Barbado Viejo, O.P. Introducción general por el R.P. Mtro. Fr. Santiago Ramírez, O.P. Tomo V. Madrid: Biblioteca de Autores Cristianos, 1954.

Scriptum super libros Sententiarum magistri Petri Lombardi episcopi Parisiensis, t. 3/2: Liber III, distinctiones XXII-XL. Parisiis: Ed.: M. F. Moos. (P. Lethielleux), 1956.

. Sententia Libri Ethicorum, cura et studio Fratrum praedicatorum, v.1. Romae: Ad Sanctae Sabinae, 1969.

WILLIAMS, T. The Franciscans. 24 p. Disponible en: < http://shell.cas.usf.edu/ - thomasw/ The Franciscans.pdf.>, Consultado el 1/06/2012.

WOLTER, A. B. Duns Scotus on Will and Morality. Edited by William Frank. Washington, D.C.: The Catholic University of America Press, 1997.

\section{REFERENCIAS DE RESPALDO}

JUAN DUNS ESCOTO. Naturaleza y voluntad: Quaestiones super libros Metaphysicorum Aristotelis, IX, q. 15. Introducción, traducción y notas de Cruz González Ayesta. Cuadernos de Anuario Filosófico Serie Universitaria 199. Pamplona: EUNSA, 2007.

Recebido em: 05.06.2012

Aceito em: 25.11.2012 
CUCCIA, E. J. 